\title{
Aerotitis: Cause, prevention, and treatment
}

\author{
S. THOMAS WESTERMAN, MD \\ MILES B. FINE, DO \\ LIANE GILBERT, MA
}

Aerotitis, an acute inflammation of the middle ear caused by the difference in air pressure between the airplane cabin and the middle-ear space, is becoming more common in the United States as our society becomes increasingly mobile. We describe a case in which a 33year-old woman with a resolving upper respiratory tract infection and mildly blocked eustachian tubes flew on a business trip. During ascent, her ears became blocked. This blockage was partially alleviated by a Valsalva's maneuver. On descent, however, her ears became severely blocked, she experienced intense pain, and her tympanic membranes ruptured. She became nauseated and vomited. Her hearing became significantly diminished and she experienced vertigo. On landing, she was taken to a local emergency room and treated with penicillin and antivertiginous medication. Subsequent otologic evaluation revealed severe permanent sensorineural hearing loss. The vestibular symptoms lasted several months. She now requires hearing aids on a permanent basis. Suggestions are presented for prevention and treatment of aerotitis.

Dr Westerman is associate professor of otolaryngology, Hahnemann University, Philadelphia. Dr Fine is currently a resident in otolaryngology/facial plastic surgery, Botsford General Hospital, Farmington Hills, Mich. Ms Gilbert is president, Westerman Research Associates, Shrewsbury, NJ.

Reprint requests to $\mathrm{S}$. Thomas Westerman, MD, 499 Broad St, Shrewsbury, NJ 07702.
Aerotitis media, or barotrauma of the middle ear, is a nonsuppurative inflammation that is usually produced when the internal pressure of the middle ear is less than that of the surrounding atmospheric pressure ${ }^{1-3}$

Aerotitis is becoming more common in the United States as our society becomes increasingly mobile. Most high-altitude passenger aircraft today fly between about 20,000 and $40,000 \mathrm{ft}$. Cabin pressure is generally maintained at the equivalent of approximately $10,000 \mathrm{ft}$, while the cruising altitude is almost four times that elevation. ${ }^{4}$ Barometric pressure at sea level is $760 \mathrm{~mm} \mathrm{Hg}$. At $20,000 \mathrm{ft}$, the pressure drops to about $350 \mathrm{~mm} \mathrm{Hg}$, and at $40,000 \mathrm{ft}$, the pressure drops to $140 \mathrm{~mm} \mathrm{Hg.}{ }^{5}$

The middle ear can tolerate and equalize air pressure changes in the lower air-pressure ranges (between 10,000 and 40,000 ft) much more easily than it can at the higher pressures found between $10,000 \mathrm{ft}$ and sea level. The pressure changes endured by the middle-ear space during flight often require rapid equilibration with the cabin environment. The eustachian tube is structured like a one-way flutter valve, allowing air to escape from the middle ear more easily than to gain entrance. For this reason, the middle-ear mechanisms cannot always maintain pressure equilibration. When this pressure differential exceeds $90 \mathrm{~mm} \mathrm{Hg}$, the eustachian tube musculature cannot open the tube. ${ }^{2}$ This is especially true on descent. Thus, the environmental air pressure remains significantly higher than the air in the middleear space. 
The middle-ear space cannot tolerate a sustained negative pressure and, when it occurs, the body attempts to equalize the pressure differential by causing serous fluid to be produced by the mucous glands in the middle ear. ${ }^{5,6}$ Sufficient negative pressure may exist to cause the rupture of blood vessels from the surrounding vasculature, resulting in a hemotypanum or, in severe cases, causing bulging and even perforation of the tympanic membrane.

On ascent, the environmental barometric pressure drops and the middle-ear pressure becomes more positive in comparison. The valvular action of the eustachian tube offers very little resistance to outward flow of air from the middle ear. Equilibration of middle-ear pressure and environmental air usually occurs, so significant aerotitis during ascent is rare. ${ }^{4}$ The pressure-equalizing ability of the eustachian tube is dependent on the rate of pressure change in the middle ear. ${ }^{7}$ The slower the pressure change, the less chance for pathologic changes to occur.

\section{Symptoms and complications}

The first symptoms an individual experiences when there is eustachian tube blockage are usually a feeling of fullness and blockage in the ears, followed by decreased hearing. Bubbling of fluid may be heard as well as felt. The pressure in the middle ear may progress to pain. In some cases, marked hearing loss, vertigo, and rupture of the tympanic membrane can occur.

The symptoms of ear blockage, pain, hearing loss, and slight vertigo are usually temporary, but permanent hearing loss, vertigo, and tinnitus may occur. A rupture of the tympanic membrane is usually self-healing but, in some cases, a tympanoplasty may be required.

There is an increased risk factor of aerotitis with the presence of middle-ear abnormalities that may cause eustachian tube dysfunction, such as swelling of the eustachian tubes caused by an upper respiratory tract illness, allergy, chronic middle-ear disease, or nasal chamber edema during pregnancy. The pressure differentials between the middle ear and the cabin pressure may also affect people who have had recent stapes procedures or tympanoplasties, especially during the healing phase in the first 3 months postoperatively. The prosthesis may become dislodged and the drum may rupture. Patients who have had stapes procedures or tympanoplasties should not fly for approximately 3 months postoperatively.

\section{Report of a case}

A 33-year-old woman with resolving upper respiratory tract infection and mildly blocked eustachian tubes flew from Chicago, Ill, to Newark, NJ, on a business trip. During ascent, her ears became more blocked, which was partially alleviated by a Valsalva's maneuver (swallowing while pinching her nose). On descent, her ear symptoms progressed from a moderate to severely blocked condition. Mild ear pain quickly progressed to severe pain binaurally, causing her to cry out. Her tympanic membranes ruptured, and a small amount of bright red blood flowed out of the external ear canals. She felt nauseated and then vomited. Her hearing became severely obtunded. On landing, she was brought to a local emergency room and treated with intravenous hydration, parenteral hydroxyzine, and penicillin. Subsequent otologic evaluation that week revealed a severe binaural sensory-neural hearing loss with mild labyrinthitis. The vestibular symptoms lasted for several months. Some hearing was regained, but most of her hearing loss was permanent, necessitating hearing aids. Her speech reception threshold was similar binaurally, averaging 70 decibels, with a speech discrimination of $60 \%$.

\section{Prevention and treatment}

Any upper respiratory tract pathologic conditions, such as an acute upper respiratory tract illness or allergy, may increase the chance of experiencing aerotitis. Persons who have had middle- or inner-ear procedures should not fly for at least 3 months postoperatively.

A traveler might consider chewing gum to allow the eustachian tube muscles to function and thereby temporarily open the eustachian tube to permit equalization of pressure. A Valsalva's maneuver will increase nasal pressure and usually is a significant aid in equalization of pressure. Swallowing water while performing the Valsalva's maneuver may further facilitate opening of the eustachian tube. Decongestion of the nasal chambers and the naso- 
pharynx with vasoconstrictors, nasal sprays, and antihistamine decongestants may all help prevent or alleviate aerotitis.

The traveler should attempt to remain awake during ascent and descent, as ear blockage can occur due to infrequency of swallowing. ${ }^{5}$ It is interesting to note that infants who are bottle fed just before and during descent have far fewer symptoms of aerotitis than nonfeeding infants. ${ }^{8}$

If a person is significantly afflicted with one of the aforementioned pathologic conditions (especially with eustachian tube blockage), and finds it essential to fly, a myringotomy (with or without a tube) can be performed to help avoid the problems associated with aerotitis. Patients with recurrent chronic aerotitis, especially those who fly frequently and whose aerotitis cannot be alleviated with medications, may also benefit from a myringotomy and tube placement.

A physician who sees a patient with acute aerotitis should treat the patient appropriately, as the situation necessitates, with antibiotics, decongestants, inflation of the eustachian tubes, either alone or in combination with myringotomy. A small middle-ear transudate can usually be successfully treated with a minimal amount of medication. Antihistamines/decongestants may be the only medications required. Middle-ear infections can occur quickly, requiring the use of antibiotics. A bulging, painful tympanic membrane can be treated with a myringotomy to both equalize the pressure and evacuate the fluid that has collected in the middle-ear space. Audiometric examination with tympanometry should be performed in patients with aerotitis to aid in the diagnosis and to follow the course of the disease.

Electronystagmography may be helpful in following a vertiginous complication. Vertigo should be treated with antivertiginous medications. Sensorineural hearing loss may be treated with vasodilators or corticoids (or both). If an inner-ear fistula has occurred, surgical intervention may be necessary.

\section{Summary}

Aerotitis, although usually temporary, may lead to permanent hearing loss, tinnitus, and vertigo. Patients with upper respiratory tract infections, allergies, and chronic middle-ear disease, and patients who have had recent ear surgeries, and even pregnant women with nasal edema, are at higher risk for aerotitis, and may need prophylactic drug therapy. Chewing gum, swallowing, decongestants, and antihistamines will help most people to overcome the pressure buildup leading to aerotitis. Ear pain, tinnitus, vertigo, or otic discharge following air travel should not be taken lightly, and an otolaryngologist should be seen immediately for evaluation.

1. Dickson E, McGibbon J, Campbell A: Acute otitic barotrauma: Clinical findings, mechanism and relationship to the pathological changes produced experimentally in the middle ears of cats by variations of pressure. J Laryngol Otol 1943;58:465-488.

2. Pulec J: Diseases of the eustachian tube, in Paparella M, Shumrick D (eds): Otolaryngology. Philadelphia, WB Saunders Co, 1980, vol 2, p 1414.

3. Armstrong H, Heim J: Effect of flight on the middle ear. JAMA 1937;109:417.

4. Campbell P: The ear, in Jackson C, Jackson C (eds): Disease of the Ear, Nose and Throat, ed 2. Philadelphia, WB Saunders Co, 1959, vol 3, p 412.

5. Hanna H: Aviation aspects of otolaryngology, in English G (ed): Otolaryngolegy. Philadelphia, JB Lippincott, 1988, vol II, p 4.

6. Raman R: Middle ear effusion: A theoretical neuromechanical hypothesis. J Laryngol Otol 1988;102:10-13.

7. Tjernstrom O, Bylander A, Groth P, et al: Eustachian tube function: Physiology and role in otitis media: Pressure chamber tests for eustachian tube function. Ann Otol Rhinol Laryngol 1985;94:Suppl 120.

8. Byers P: Infant crying during aircraft descent. Nurs Res 1986;35:260-262. 